\title{
Can hidden correlations mimic a variable fine structure constant?
}

\author{
R. Bandiera ${ }^{1}$ and E. Corbelli ${ }^{2}$ \\ 1 INAF - Osservatorio Astrofisico di Arcetri, Largo E. Fermi 5, 50125 Firenze, Italy \\ e-mail: bandiera@arcetri.astro.it \\ 2 INAF - Osservatorio Astrofisico di Arcetri, Largo E. Fermi 5, 50125 Firenze, Italy \\ e-mail: edvige@arcetri.astro.it
}

Received 27 July 2004 / Accepted 20 December 2004

\begin{abstract}
Murphy et al. (2003a, MNRAS, 345, 609) claim to find evidence of cosmological variations of the fine structure constant $\alpha$ in the spectra of intervening QSO absorption line systems. We find that this result is affected by systematic effects. The $\alpha$ values estimated in individual line systems depend on the set of atomic transitions used and therefore the quoted dependence on the cosmic age may reflect the fact that different sets of transitions are used at different redshifts. A correlation between line shifts and relative optical depths of the atomic transitions is also present. This correlation is very tight for a high-redshift subsample and accounts for the anomalous dispersion of the $\alpha$ values found by Murphy et al. (2003a) in this subsample. The above correlations are consistent with a scenario in which gravitational redshift, caused by the gravity of the dark halo, contributes to the shift in frequency of individual components. Gravitational redshift causes differential spectral shifts of the same order as magnitude of those measured. In the presence of line misidentification, these shifts can be interpreted in terms of a variable $\alpha$. In order to verify the gravitational redshift hypothesis, a direct access to Murphy et al. (2003a) data, or to a large amount of new high resolution data, is necessary.
\end{abstract}

Key words. quasars: absorption lines - galaxies: halos - cosmology: miscellaneous

\section{Introduction}

The hypothesis of a cosmological evolution of the fine structure constant $\alpha$ has been investigated in a series of papers (Murphy et al. 2003a; Webb et al. 2003; Murphy et al. 2001a; Webb et al. 2001, 1999, also collectively referred to as MWF), by applying a sophisticated spectral analysis, called the "many multiplet" method (hereafter MM), to large samples of absorption line systems in QSO spectra. This method is based on a $\chi^{2}$ analysis of multiple-component profile fits in several transitions, and relies on theoretical calculations of how the relative positions of different transitions change due to $\alpha$ variations (e.g. Dzuba et al. 2002). The use of many transitions improves the accuracy of $\alpha$ determination in each absorption system, compared to fits of only two transitions (like the alkali-doublet method, e.g. Murphy et al. 2001c). In addition, the use of large samples entails a much smaller quoted uncertainty on the average value of $\Delta \alpha / \alpha$ (up to 128 absorption systems were analyzed in Murphy et al. 2003a). The conclusion of MWF is that the value $\alpha$ was slightly smaller at earlier cosmological epochs $\left(\Delta \alpha / \alpha \sim-0.5 \times 10^{-5}\right.$, with a $5-\sigma$ significance). Ancillary papers (Murphy et al. 2001b, 2003b) have reached negative conclusions on potential systematic effects which could mimic a variable fine structure constant (hereafter VFSC).

A recent work by Chand et al. (2004), based on more accurate measurements in high quality data relative to a smaller sample objects, uses the MM method with well-defined selection criteria. No cosmological variation of $\Delta \alpha / \alpha$ is found, with a $1-\sigma$ uncertainty of $0.06 \times 10^{-5}$ (namely an order of magnitude below the values quoted by MWF). Chand et al. (2004) avoid very weak and blended lines and point out that when one does not do so, spurious detections are frequently seen. Using a different but very robust method, Bahcall et al. (2004) also do not find any relevant time dependence of the fine structure constant. Bahcall et al. (2004) point out several inconsistencies in the results of MWF which imply that systematic uncertainties due to misidentification of lines might be significant in the MM method used by MWF. In order to directly test the hypothesis of component misidentifications, one needs to investigate in detail the original data and the routines used by MWF in their analysis: this information is, however, not accessible to the community. As emphasized by Chand et al. (2004) and by Bahcall et al. (2004), MWF have not described their algorithm of line identification, the confidence level adopted and the physical assumptions made. So, line misidentifications cannot be excluded in their results.

Given the overwhelming consequences in the fundamental physics and cosmology of the variability of the fine structure constant (see e.g. Martins 2003; Uzan 2003, 2004), any newly suggested potential source of bias in the determination of $\Delta \alpha / \alpha$ must be examined in detail. Direct access to MWF data and analysis would facilitate this process. At the moment, however, we are forced to approach the problem in different ways. In the 
present paper we investigate the statistical properties of the data presented by Murphy et al. (2003a, their Table 3), and we find correlations that can be explained only in terms of a systematic effect, not accounted for by MWF (Sect. 2). In Sect. 3, we put forward the hypothesis that differential gravitational redshift (hereafter GRS), within absorption systems, may be responsible for the correlations found and of the non-zero value of $\Delta \alpha / \alpha$. An important feature of GRS, with respect to other effects, is that symmetry between red- and blueshifts is broken, and therefore a systematic effect on the average values of the spectral shifts may be accounted for. This effect is very tiny, and it can be detected only under particular conditions. In the MM analysis, GRS appears in only if misidentifications of some components are present. In Sect. 4, we search for correlations between $\alpha$ and some indicator of the gravitational potential, for various absorption systems: the presence of such correlations represents a strong clue that some differential redshift is of gravitational origin. Our last section summarizes and concludes that GRS is a possible candidate for the origin of the measured differential redshift.

\section{A statistical analysis of many-multiplet method results}

The central working hypothesis of MWF for evaluating $\Delta \alpha / \alpha$ is the proportionality between the line shift and the atomic relativistic correction for all atomic transitions detected in the spectrum of each component of an absorption system. In the framework of the MM method, one expects the following relation to hold for each atomic transition $i$ :

$$
\frac{\Delta v_{i}}{v_{i}}=Q_{i} \frac{\Delta \alpha}{\alpha}+K_{z}
$$

where $\Delta v_{i} / v_{i}$ are the line shifts measured for a specific component of the absorption system, with their intrinsic sign. The coefficients $Q_{i}$ are given by the ratio $q_{i} / \omega_{i}$, where $\omega_{i}$ is the transition wavenumber, and $q_{i}$ is the atomic relativistic correction (see Table 2 of Murphy et al. 2003a), and $K_{z}$ is the redshift of that specific component. If $\Delta v_{i} / v_{i}$ and $Q_{i}$ are correlated, MWF analysis derives a value of $\Delta \alpha / \alpha$ different from zero.

We start with the working hypothesis that there is a primary correlation between the relative optical depth of a transition and $\Delta v_{i} / v_{i}$, i.e. that the primary correlation is not that between $Q_{i}$ and $\Delta v_{i} / v_{i}$. A preliminary step is to estimate, for a "typical" absorber, the relative optical depths of all transitions used by MWF. We define the relative optical depth of the $i$ th transition, $\tau_{i}$, as the optical depth scaled to that of a reference transition (from now on we skip, for simplicity, the index $i$ ). In this paper we will use Fe II at $2382 \AA$ as the reference transition. We take a sample of low- $z$ absorbers (Churchill 1997; Churchill \& Vogt 2001) to determine the column density of Mg I and Mg II relative to that of Fe II , and a sample of high- $z$ absorbers (Prochaska \& Wolfe 1999; Prochaska et al. 2001) to determine the column density of Al II , Al III , Si II , Cr II , Ni II , and $\mathrm{Zn}$ II relative to that of Fe II. We have linked the low and high- $z$ sets of data to derive the relative optical depths of all transitions of interest. Table 1 gives the decimal logarithm of the relative optical depth, $\log \tau$, the $\log$ arithm of the relative
Table 1. Atomic data.

\begin{tabular}{|c|c|c|c|c|c|}
\hline Ion & $\log N_{\text {rel }}$ & Wavelength & $f \times 10^{3}$ & $\log \tau$ & $Q \times 10^{2}$ \\
\hline $\operatorname{Mg} I$ & -1.6520 & $2852.96 \AA$ & 1810.0 & -0.8213 & 0.2454 \\
\hline \multirow[t]{2}{*}{ Mg II } & 0.3372 & $2796.35 \AA$ & 612.3 & 0.6885 & 0.5900 \\
\hline & & $2803.53 \AA$ & 305.4 & 0.3876 & 0.3364 \\
\hline $\mathrm{Al}$ II & -1.0454 & $1670.79 \AA$ & 1880.0 & -0.4306 & 0.4511 \\
\hline \multirow[t]{2}{*}{ Al III } & -1.5818 & $1854.72 \AA$ & 539.0 & -1.4641 & 0.8606 \\
\hline & & $1862.79 \AA$ & 268.0 & -1.7657 & 0.4024 \\
\hline \multirow[t]{2}{*}{ Si II } & 0.4659 & $1526.71 \AA$ & 127.0 & -0.1288 & 0.1038 \\
\hline & & $1808.01 \AA$ & 2.18 & -1.8207 & 0.9601 \\
\hline \multirow[t]{3}{*}{ Cr II } & -1.6784 & $2056.26 \AA$ & 105.0 & -2.2263 & -2.2763 \\
\hline & & $2062.24 \AA$ & 78.0 & -2.3542 & -2.5799 \\
\hline & & $2066.16 \AA$ & 51.5 & -2.5336 & -2.7563 \\
\hline \multirow[t]{7}{*}{$\mathrm{Fe}$ II } & 0.0000 & $1608.45 \AA$ & 58.0 & -0.9124 & -1.9301 \\
\hline & & $1611.20 \AA$ & 1.36 & -2.5415 & 1.6918 \\
\hline & & $2344.21 \AA$ & 114.0 & -0.4553 & 2.9396 \\
\hline & & $2374.46 \AA$ & 31.3 & -1.0111 & 3.8941 \\
\hline & & $2382.76 \AA$ & 320.0 & 0.0000 & 3.5694 \\
\hline & & $2586.65 \AA$ & 69.1 & -0.6295 & 3.9317 \\
\hline & & $2600.17 \AA$ & 239.0 & -0.0892 & 3.5259 \\
\hline \multirow[t]{3}{*}{ Ni II } & -1.4368 & $1709.60 \AA$ & 32.4 & -2.5756 & -0.0342 \\
\hline & & $1741.55 \AA$ & 42.7 & -2.4477 & -2.4382 \\
\hline & & $1751.92 \AA$ & 27.7 & -2.6331 & -1.2263 \\
\hline \multirow[t]{2}{*}{ Zn II } & -2.4081 & $2026.14 \AA$ & 489.0 & -2.2943 & 5.0228 \\
\hline & & $2062.66 \AA$ & 256.0 & -2.5676 & 3.2528 \\
\hline
\end{tabular}

column density, $\log N_{\text {rel }}$, the $Q$ coefficient, and some other basic data (ionic species, wavelength, and oscillator strength $f$, as given by Prochaska et al. 2001) for all transitions used.

We define the standard deviation in $\log \tau$, and the linear correlation between $\log \tau$ and $Q$ as:

$$
\begin{aligned}
& s=\sqrt{\frac{\sum\left(\log \tau-\langle\log \tau\rangle_{\mathrm{obs}}\right)^{2}}{n_{\mathrm{obs}}-1}}, \\
& \lambda=\frac{\sum\left(Q-\langle Q\rangle_{\mathrm{obs}}\right)\left(\log \tau-\langle\log \tau\rangle_{\mathrm{obs}}\right)}{\sqrt{\sum\left(Q-\langle Q\rangle_{\mathrm{obs}}\right)^{2}} \sqrt{\sum\left(\log \tau-\langle\log \tau\rangle_{\mathrm{obs}}\right)^{2}}},
\end{aligned}
$$

where the sums are performed only over the $n_{\text {obs }}$ transitions in which a given absorption system has been observed, and that have been used to derive $\Delta \alpha / \alpha$ for that absorption system (see Table 3 in Murphy et al. 2003a, for the sets of transitions used). Also the averaged values $\langle\log \tau\rangle_{\text {obs }}$ and $\langle Q\rangle_{\text {obs }}$ are computed using only the $n_{\mathrm{obs}}$ transitions that have been observed.

The values of $s$ and $\lambda$ may change from one absorption system to another, but there are absorption systems with equal values of $s$ and $\lambda$. In principle, spectral lines should be weighted with their specific relevance to the fit; however, in our simplified analysis we assume that all transitions used for an absorption system are equally relevant. The use of $\log \tau$, instead of $\tau$, has the advantage of limiting the dispersion, even though the resulting correlations are qualitatively similar to those obtained using $\tau$. If $\log \tau$ correlates with $Q$, the primary correlation between $\log \tau$ and the measured $\Delta v / v$ induces a spurious correlation between $Q$ and $\Delta v / v$. In the MWF analysis this leads to a $\Delta \alpha / \alpha$ different from zero.

Our complete sample is that listed in Table 3 of Murphy et al. (2003a). We subdivide this into low- $z$ and high- $z$ 
Table 2. High-contrast absorption line systems.

\begin{tabular}{ccrcl}
\hline \hline QSO & $z_{\text {abs }}$ & $\Delta \alpha / \alpha\left(10^{-5}\right)$ & $\log N(\mathrm{HI})$ & Ref. \\
\hline $0100+13$ & 2.310 & $-3.941 \pm 1.368$ & 21.40 & (PW99) \\
$0149+33$ & 2.140 & $-5.112 \pm 2.118$ & 20.50 & (PW99) \\
$0201+37$ & 1.955 & $1.989 \pm 1.048$ & 20.20 & (PW96) \\
$0201+37$ & 2.462 & $0.572 \pm 1.719$ & 20.40 & (PW96) \\
$0528-25$ & 2.141 & $-0.853 \pm 0.880$ & 20.70 & (L96) \\
$0528-25$ & 2.811 & $0.850 \pm 0.846$ & 21.20 & (L96) \\
$0841+12$ & 2.374 & $2.277 \pm 3.816$ & 20.95 & (PW99) \\
$0841+12$ & 2.374 & $1.435 \pm 1.227$ & 20.95 & (PW99) \\
$0841+12$ & 2.476 & $-4.304 \pm 1.944$ & 20.78 & (PW99) \\
$1011+43$ & 2.959 & $2.475 \pm 1.706$ & $\ldots .$. & (C02) \\
$1215+33$ & 1.999 & $5.648 \pm 3.131$ & 20.95 & (PW99) \\
$1759+75$ & 2.625 & $-0.750 \pm 1.387$ & 20.80 & (P01) \\
$1759+75$ & 2.910 & $-0.492 \pm 1.645$ & 19.80 & (O99) \\
$1850+40$ & 1.990 & $-1.663 \pm 0.859$ & $\ldots .$. & (C02) \\
$2206-20$ & 1.920 & $1.878 \pm 0.702$ & 20.65 & (PW97) \\
$2230+02$ & 1.859 & $-5.407 \pm 1.179$ & $<20.85$ & (PW99) \\
$2230+02$ & 1.864 & $-0.998 \pm 0.492$ & 20.85 & (PW99) \\
$2231-00$ & 2.065 & $-2.604 \pm 1.015$ & 20.56 & (PW99) \\
$2231-00$ & 2.065 & $1.707 \pm 1.249$ & 20.56 & (PW99) \\
$2231-00$ & 2.653 & $-3.348 \pm 1.904$ & $<20.30$ & (L91) \\
$2343+12$ & 2.430 & $-1.224 \pm 0.389$ & 20.34 & (L98) \\
$2359-02$ & 2.095 & $-0.068 \pm 0.722$ & 20.70 & (PW99) \\
\hline
\end{tabular}

(L91) Lanzetta et al. (1991); (L96) Lu et al. (1996); (L98) Lu et al. (1998); (O99) Outram et al. (1999); (PW96) Prochaska \& Wolfe (1996); (PW97) Prochaska \& Wolfe (1997); (PW99) Prochaska \& Wolfe (1999); (P01) Prochaska et al. (2001); (C02) Currant et al. (2002).

subsamples, using a spectroscopic criterion: an absorber is included in the high- $z$ sample only if it has been observed in at least one transition at wavelengths shorter than $2300 \AA$. Murphy et al. (2003a) have noticed that a subsample of systems (called the "high-contrast sample"), observed in many transitions with very different relative optical depths, has a statistical spread in $\Delta \alpha / \alpha$ much larger than the average nominal uncertainty of each absorber. The authors ascribe this discrepancy to uncertainties not accounted for in their analysis, but they exclude that this may lead to systematic effects in the determination of $\Delta \alpha / \alpha$. We shall reach a different conclusion.

Figure 1 shows the complete set of data in the $s-z$ plane, and in the $s-\lambda$ plane. Figure 1a shows that our criterion, although purely based on the set of transitions used, effectively separates lower and higher- $z$ absorbers, with a shallow cut around $z \sim$ 1.8. Values of $s$ for low $z$ absorbers are limited to the range $(0.3,0.7)$; values of $s$ for high- $z$ objects lie in the range $(0.3$, 1.2). High-contrast absorption systems typically correspond to higher values of $s$ (say, above 0.8 ): this indicates that, at least on average, the quantity $s$ accounts reasonably well for the spread in relative optical depth of the atomic transitions used.

Because different sets of transitions are used for different redshifts, any cosmological implication of measured variations of $\Delta \alpha / \alpha$ with redshift should be taken very cautiously. Notice that, in addition, the low $z$ and high- $z$ samples given by Murphy et al. (2003a) correspond to different kinds of absorbers. The low- $z$ sample is mostly composed of absorption-line systems

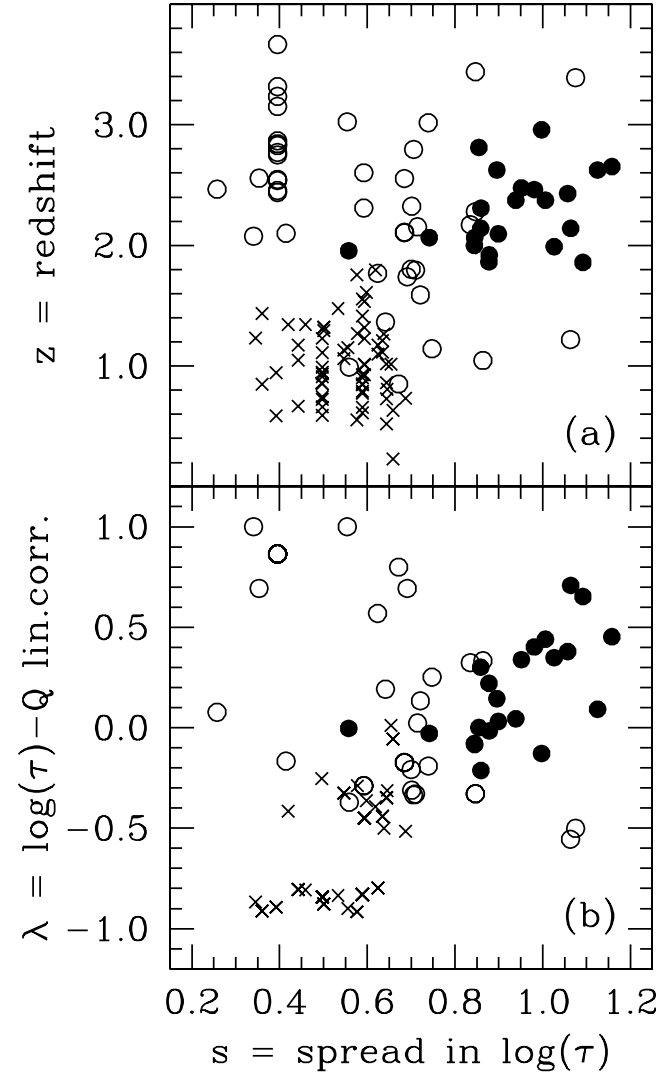

Fig. 1. a) Distribution of the data in the $s-z$ parameter plane and b) in the $s-\lambda$ plane. Crosses are for objects in the low- $z$ sample and circles for the high- $z$ sample (filled circles indicate objects in the "highcontrast" subsample). In b) the number of points is smaller than in a), because in b) a single mark may indicate more than one absorption system.

selected by the Mg II lines, and have moderate HI column densities; while a large fraction of the high- $z$ sample is made up of damped Ly $\alpha$ absorption systems. Therefore, we should not be surprised if, in our analysis, different results are found for the two sets of systems, since they have been analyzed in different sets of transitions, and furthermore are associated with different types of cosmic structures.

In the two subsections below we will search for whether individual values of $\Delta \alpha / \alpha$ depend on $\lambda$. A significant correlation would prove that our working hypothesis is correct; if instead the MWF results correspond to a true variation of $\alpha$ no correlation should be found. For $\lambda$ positive, the (spurious) correlation between $\Delta v / v$ and $Q$ will have the same sign as the primary correlation between $\Delta v / v$ and $\log \tau$. For $\lambda$ negative the correlation will have the opposite sign. In order to obtain a negative $\Delta \alpha / \alpha$ when $\lambda$ is positive, $\Delta v / v$ must decrease for increasing $\log \tau$. When $\lambda$ is negative $\Delta v / v$ must increase for increasing $\log \tau$. According to our working hypothesis, the closer the $\lambda$ value is to \pm 1 , the larger the displacement from zero of the $\Delta \alpha / \alpha$ value for each system. Therefore we expect a correlation between $\Delta \alpha / \alpha$ and $\lambda$. The average value of $\Delta \alpha / \alpha$ over the whole sample will be different from zero, provided that the $\lambda$ distribution is not symmetric around zero. 


\subsection{Low-z sample}

Figure $1 \mathrm{~b}$ shows that negative values of $\lambda$ are mostly associated with low- $z$ objects (64 objects). A weighted linear regression between $\Delta \alpha / \alpha$ and $\lambda$ results in a positive slope, although at a low significance level: $m=(0.80 \pm 0.60) \times 10^{-5}$, with a reduced $\chi^{2}$ of 1.10 (assuming a functional dependence $\Delta \alpha / \alpha=m \lambda+$ $q)$. A tighter result $(4.5 \sigma)$ is obtained assuming a functional dependence $\Delta \alpha / \alpha=m_{0} \lambda: m_{0}=(0.92 \pm 0.20) \times 10^{-5}$, with a reduced $\chi^{2}$ of 1.09. Below we again will use the symbols $m$ and $m_{0}$ to indicate the best fitting slope leaving $q$ free, or setting $q=0$.

As can be seen in Fig. 1b, low- $z$ objects present a bimodal distribution, consisting of one component with $\lambda$ in the range $(-0.92,-0.80)$, and of another one with $\lambda$ in the range $(-0.52,0.01)$. The former component is highly uniform in $\lambda$ (39 objects with an average $\lambda$ value of -0.85 ) and the average $\Delta \alpha / \alpha$ is $(-0.80 \pm 0.18) \times 10^{-5}$. For the other component (25 objects with an average $\lambda$ value -0.35 ) we have instead $\Delta \alpha / \alpha=(-0.36 \pm 0.23) \times 10^{-5}$. A comparison of the two subsamples again suggests that $\Delta \alpha / \alpha$ increases with $\lambda$. However, as already obtained from the linear regression, the significance of this trend becomes compelling only if we assume that $\Delta \alpha / \alpha$ vanishes when $\lambda=0$.

It is worth investigating another recently published work (Chand et al. 2004), which analyzes the spectra of a sample of low- $z$ absorption systems. The authors do not find any evidence of a VFSC and give an upper limit of $0.06 \times 10^{-5}$ to $\Delta \alpha / \alpha$. This is about one order of magnitude smaller than the signal claimed by MWF. The sample of Chand et al. (2004) is smaller than that of Murphy et al. (2003a) but the quoted errors of $\Delta \alpha / \alpha$ are smaller because high resolution observations allowed them to select only systems with all individual components identified unambiguously. The decision of applying a severe selection of the systems could either render the approach cleaner than that of MWF, or potentially more subject to biases. Here we check whether the correlation between $\Delta \alpha / \alpha$ and $\lambda$, found in the MWF low- $z$ sample, is also present.

Values of $\lambda$ in Chand et al. (2004) were obtained using all transitions listed for each of the 23 absorption systems in their Table 3 (even if some components were analyzed by Chand et al. (2004) using a subsample of these transitions). The estimated slope for the $\lambda-\Delta \alpha / \alpha$ linear regression is $m=$ $(0.15 \pm 0.14) \times 10^{-5}$, with a reduced $\chi^{2}$ of 0.94 ; in the case of a pure linear proportionality we have $m_{0}=(0.12 \pm 0.08) \times 10^{-5}$, with a reduced $\chi^{2}$ of 0.91 . This result is consistent with no correlation between $\lambda$ and $\Delta \alpha / \alpha$, down to a 1.1-1.5 $\sigma$ level, namely at a level 5-8 times smaller than the correlation found in the Murphy et al. (2003a) results. We have also used different methods of object selection, such as limiting our choice to systems in which both $\mathrm{Fe}$ and $\mathrm{Mg}$ transitions are detected in all components (12 objects) or to systems in which the same set of transitions has been used for all components ( 8 objects). The conclusion, to a rather high level of confidence, is that the correlation is not visible in the Chand et al. (2004) results. Their method does not seem to be affected by the unwanted systematic effects found in Murphy et al. (2003a).

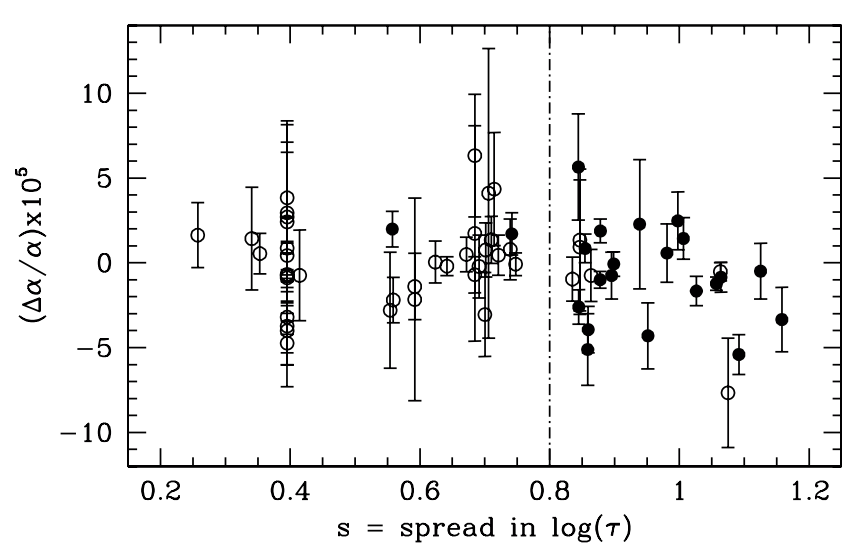

Fig. 2. Dependence on the spread in the relative optical depths $s$ of the $\Delta \alpha / \alpha$ values, for the high- $z$ sample. The dot-dashed line divides the figure in two parts: for $s<0.8$ no trend of $\Delta \alpha / \alpha$ with $s$ is apparent; for $s>0.8$, instead, values of $\Delta \alpha / \alpha$ decrease for increasing $s$. As in Fig. 1, filled circles indicate the "high-contrast" subsample.

\subsection{High-z sample}

Figure $1 b$ shows that high- $z$ absorbers are distributed more continuously over a wider range of $\lambda$, namely $(-0.6,+1.0)$. With respect to the low $-z$ sample, the high- $z$ sample shows a much larger dispersion in $s$ (see Fig. 1), and therefore it may be better suited for searching for effects which arise when using transitions with a wide range of relative optical depths (i.e. cases with large $s$ values). Figure 2 shows how the measured values of $\Delta \alpha / \alpha$ depend on $s$ in this sample. While no apparent trend is present for $s<0.8$, for larger $s$ values there is a clear trend of decreasing $\Delta \alpha / \alpha$ with $s$. A difference between these two subsamples is present also in the measured values of $\Delta \alpha / \alpha$ : the average of $\Delta \alpha / \alpha$ for $s<0.8$ (38 objects) is $(-0.04 \pm 0.24) \times 10^{-5}$, for $s>0.8$ (26 objects) is $(-0.82 \pm 0.19) \times 10^{-5}$. The discrepancy between these two values is at a $2.6 \sigma$ level. A result similar to the $s>0.8$ sample, namely $\Delta \alpha / \alpha=(-0.68 \pm 0.20) \times 10^{-5}$, is obtained for the high-contrast sample: this is a rather obvious result, since the two samples are partially overlapping. In the remaining part of this section, we concentrate on a statistical analysis of the high-contrast subsample.

The high-contrast sample is made of 22 objects for which Murphy et al. (2003a) have measured an excess dispersion of $\Delta \alpha / \alpha$. We propose that this excess dispersion results from the presence of hidden variables, one of which is $\lambda$. A simple way to derive how $\Delta \alpha / \alpha$ varies with $\lambda$ is to evaluate the average $\Delta \alpha / \alpha$ for systems with negative and positive $\lambda$ : we obtain $\Delta \alpha / \alpha$ equal to $(+0.63 \pm 0.43) \times 10^{-5}$ and $(-1.04 \pm 0.22) \times 10^{-5}$ respectively, two values which are $3.5 \sigma$ apart. A weighted linear regression between $\Delta \alpha / \alpha$ and $\lambda$ on the high-contrast sample gives a slope $m=(-3.46 \pm 0.88) \times 10^{-5}(3.9 \sigma$ level $)$. Assuming that $\Delta \alpha / \alpha$ vanishes at $\lambda=0$, the slope is $m_{0}=(-3.21 \pm 0.62) \times 10^{-5}$ $(5.2 \sigma$ level $)$.

In spite of the good significance level of $\Delta \alpha / \alpha-\lambda$ regressions for the high-contrast sample, their reduced $\chi^{2}$ values are still large, of the order of 3. A possibility is that the Murphy et al. (2003a) hypothesis applies mostly to heavily damped systems, because they are complex systems, containing many saturated lines, while our conjecture of the presence of hidden 


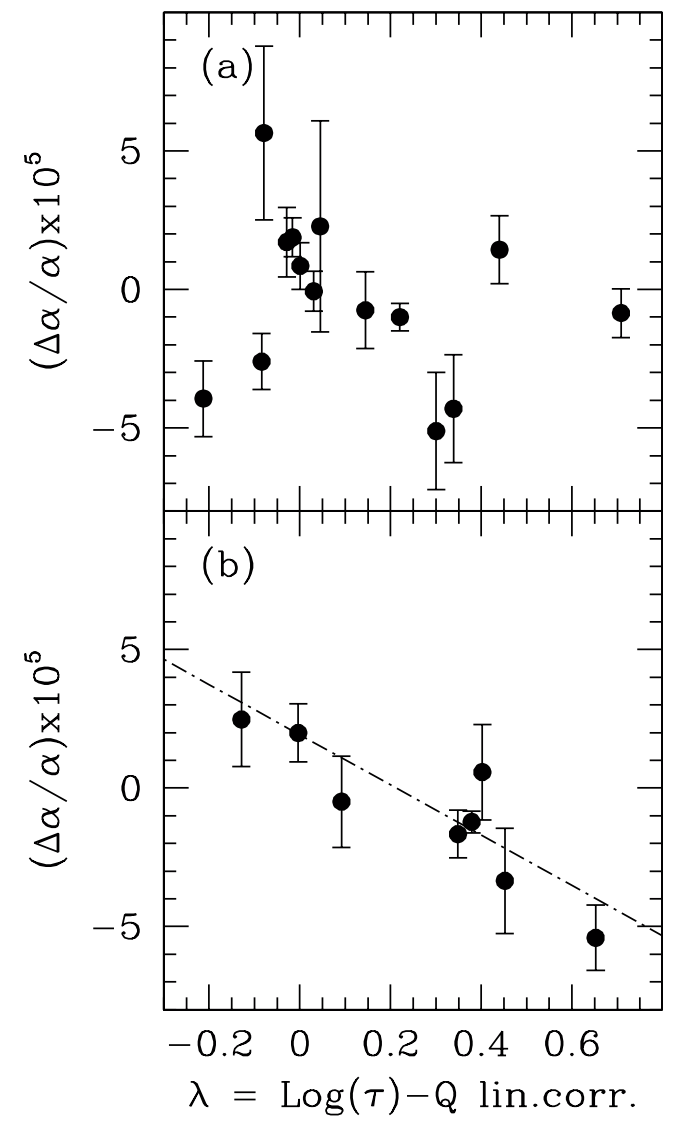

Fig. 3. Correlations between $\Delta \alpha / \alpha$ and $\lambda$ for high-contrast (high-z) objects, a) $N(\mathrm{H} \mathrm{I})>3 \times 10^{20} \mathrm{~cm}^{-2}$, b) $N(\mathrm{H} \mathrm{I}) \leq 3 \times 10^{20} \mathrm{~cm}^{-2}$.

variables may better apply to absorption systems below a critical column density, $N_{\mathrm{c}}$.

In Table 2 we list all high-contrast systems, and we indicate the H I column density whenever this can be found in the literature (the relative references are listed in the last column). For a few cases the H I column density is unknown: we assume for those that the H I column density is below $N_{\mathrm{c}}$. In Fig. 3a we show the data of Table 2 for $N(\mathrm{HI})>N_{\mathrm{c}}=3 \times 10^{20} \mathrm{~cm}^{-2}$. A weighted linear regression for these systems gives a slope $m=(-1.08 \pm 1.15) \times 10^{-5}$ (with a reduced $\chi^{2}$ of 3.62); or alternatively $m_{0}=(-1.42 \pm 0.97) \times 10^{-5}$ (with a reduced $\chi^{2}$ of 3.37). Therefore there is no evidence of correlation in this subsample. For $N(\mathrm{HI}) \leq N_{\mathrm{c}}=3 \times 10^{20} \mathrm{~cm}^{-2}$ instead a weighted linear regression gives a slope $m=(-9.08 \pm 1.85) \times 10^{-5}$ (with a reduced $\chi^{2}$ of 0.91$)$; Fig. $3 \mathrm{~b}$ displays this result. Alternatively, $m_{0}=(-4.46 \pm 0.81) \times 10^{-5}$ (with a reduced $\chi^{2}$ of 1.87$)$. Here both regressions determine the slope at a significance level of about $5 \sigma$. The result we find is striking: the correlation between $\Delta \alpha / \alpha$ and $\lambda$ is very tight for low column density systems and it loses its significance for heavily damped systems. One worry about choosing $N_{\mathrm{c}}$ as low as $3 \times 10^{20} \mathrm{~cm}^{-2}$ might be the 3 unknown H I column densities of Table 2 . These can turn out to be higher than $3 \times 10^{20} \mathrm{~cm}^{-2}$. However, we have checked that as soon as $N_{\mathrm{c}} \leq 7 \times 10^{20} \mathrm{~cm}^{-2}$ the significance of the correlation stays above $4.5 \sigma$ for $N(\mathrm{HI}) \leq N_{\mathrm{c}}$ (even though the dispersion increases) and stays below $1 \sigma$ for $N(\mathrm{HI})>N_{\mathrm{c}}$.
For $N_{\mathrm{c}}=7 \times 10^{20} \mathrm{~cm}^{-2}$ for example, our last two statements are true not only if all the unknown $\mathrm{HI}$ column densities are below $N_{\mathrm{c}}$ but also if we do not consider for the correlations the two entries relative to the $(\mathrm{C} 02)$ reference of Table 2, or if we consider one or both of them to be higher than $N_{\mathrm{c}}$.

\section{Differential gravitational redshift in absorption systems}

In this section we discuss GRS in absorption systems, and its possible influence on the quoted variation of the variable fine structure constant. GRS can be detected in many types of bound systems such as stars, galaxies and clusters of galaxies (e.g. Broadhurst \& Scannapieco 2000; Stiavelli \& Setti 1993), and has been used to infer physical parameters like the total mass. It is likely that absorption systems are localized in dark matter halos of galaxies, but since they can be observed only along one line of sight, whose position with respect to the system barycentre is unknown, it is not easy to quantify the magnitude of GRS in observable quantities. To check explicitly if and how GRS plays a role in the MWF analysis is even more difficult because their data and procedure are not available to the public.

In order for GRS to be responsible for the fine structure constant variations measured in absorption line systems with the MM method, three conditions are required:

i) the differential GRS between the innermost regions and the outskirts of typical absorption systems should be at least of the same order as the relative shifts measured by MWF (of the order of $0.1 \mathrm{~km} \mathrm{~s}^{-1}$, and interpreted in terms of a VFSC);

ii) misidentification of individual components in a line profile should affect the MM analysis, as carried on by MWF, i.e. components attributed to the same cloud in different transitions are instead associated with different clouds;

iii) the kinematics should allow a detection of a tiny spectral shift of gravitational origin.

In the rest of this section we examine these conditions in more detail. In Sect. 4, we test in a more quantitative way the GRS hypothesis, by searching for correlations between the estimated $\Delta \alpha / \alpha$ and indicators of the gravitational potential in the absorption system.

\subsection{Galactic haloes and gravitational redshift}

The aim of this subsection is to evaluate, for typical gaseous systems embedded in dark matter haloes, the magnitude of the differential GRS. Photons of frequency $v_{0}$ emitted in a gravitational potential $\Phi$ are observed at infinity to be redshifted by an amount

$\frac{\Delta v}{v_{0}}=\frac{\Phi}{c^{2}}$

where $\Delta v=v-v_{0}$, and $c$ is the speed of light.

Let us consider gaseous systems embedded in dark matter haloes whose density profile is described by the formula proposed by Navarro et al. (1996, 1997):

$\rho(R)=\frac{\rho_{\mathrm{NFW}}}{\left(R / R_{\mathrm{NFW}}\right)\left(1+R / R_{\mathrm{NFW}}\right)^{2}}$. 
If $R_{\Delta}$ is the radius of a sphere containing a mean density $\Delta$ times the critical density at redshift $z$, and $M_{\Delta}$ is the mass inside $R_{\Delta}$, one can express the gravitational potential of a dark matter halo at a redshift $z$ in terms of $M_{\Delta}$ and of the concentration parameter $C_{\Delta} \equiv R_{\Delta} / R_{\mathrm{NFW}}$ (Navarro et al. 1996, 1997). Numerical simulations of structure formation often reveal a correlation between $C_{\Delta}$ and $M_{\Delta}$ which depends on the details of the assumed cosmological model (e.g. Navarro et al. 1996, 1997; Avila-Reese et al. 2001; Eke et al. 2001). For a Cold Dark Matter-dominated universe, the dependence of $C_{\Delta}$ on $M_{\Delta}$ is weak. We consider $\Delta=200$, as originally proposed by Navarro et al. $(1996,1997)$, and define $C \equiv C_{200}$. Here we do not assume any explicit cosmological model to relate $C$ and $M_{200}$ but use $C \simeq 2-10$ at redshifts $0.5-3$, as suggested by numerical simulations of dark matter haloes forming in a Cold Dark Matterdominated universe. The gravitationally redshifted frequency of an absorption line inside a galactic potential dominated by dark matter can therefore be written as:

$$
\begin{aligned}
\frac{\Delta v}{v_{0}} & =-\frac{G M_{200}}{4 \pi c^{2} R_{200}} \frac{\ln (1+C x)}{x\{\ln (1+C)-C /(1+C)\}} \\
& \simeq-\frac{2.3 \times 10^{-8}}{\left(\Omega_{0} \Omega\right)^{1 / 3}}\left(\frac{M_{200} / h^{-1}}{10^{12} M_{\odot}}\right)^{2 / 3} \frac{(1+z) \ln (1+C x)}{x\{\ln (1+C)-C /(1+C)\}}
\end{aligned}
$$

where $G$ is the gravitational constant, $\Omega$ is the matter density of the Universe at $z$ and we have introduced the variable $x \equiv R / R_{200}$. In Fig. 4 we show the GRS in $\mathrm{km} \mathrm{s}^{-1}$ as a function of the galactocentric distance $x$ at $z=2$ for $\Omega_{0} / \Omega \simeq 1$. For a flat, cosmological constant dominated universe with $\Omega_{0}=0.3$, $\Lambda_{0}=0.7$, the GRS is a factor $\sim 1.5$ larger than what Fig. 4 shows. There are no noticeable differences in the magnitude of the GRS if we consider other gravitational potentials with similar masses but no cusp in the centre. For a Burkert potential (Burkert 1995), for example, we find less than a factor 2 difference over the whole range of masses and radii shown in Fig. 4.

An interesting result of Fig. 4 is that the velocity shifts are of the same order of magnitude as those found in the analysis of Murphy et al. (2003a, see their Table 4) and used to claim a VFSC. The estimate of differential GRS presented above refers to the whole absorption system; differential GRS within individual clouds (detected as individual components in the line profile) is much smaller.

\subsection{Detectability of GRS in QSO absorption systems}

We outline here the most appropriate conditions to detect GRS effects in QSO absorption systems. In principle, GRS could be detected using even a single transition, if the available sample of absorption lines is large enough to justify a statistical approach. In fact, isotropy arguments show that, in the absence of GRS, kinematic redshifts of individual spectral components should average to zero with a symmetric distribution in the whole sample. GRS, instead, induces skewness in the spectral distribution of the absorption components, because absorption lines located more deeply in the potential well are those more strongly redshifted. In this kind of analysis, the main practical difficulty is to cope with the smallness of this effect, compared to both instrumental and statistical uncertainties. Even in

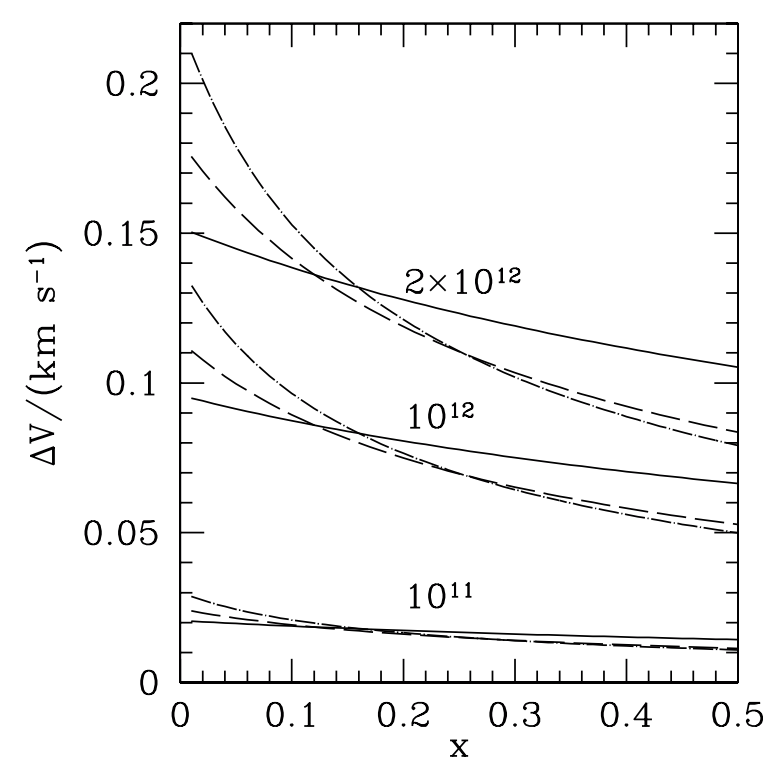

Fig. 4. GRS in $\mathrm{km} \mathrm{s}^{-1}$ as a function of the galactocentric distance $x=R / R_{200}$ at $z=2$. The 3 sets of curves correspond to the halo masses $M_{200} h=10^{11}, 10^{12}, 2 \times 10^{12} M_{\odot}$. The continuous lines are for concentration values $C=2$, the dashed lines for $C=6$, the dashdotted lines for $C=10$.

the absence of instrumental uncertainties, there is a minimum number of spectral components below which it is impossible to extract the gravitational shift from the statistical dispersion due to kinematic motion. The problem is qualitatively similar to that of determining, with accuracy $\Delta V_{\sigma}$, the average shift for a sample of components whose distribution shows a dispersion $\Delta V_{ \pm}$: in this case at least $\sim\left(\Delta V_{ \pm} / \Delta V_{\sigma}\right)^{2}$ different components are required. If we aim at detecting $\Delta V_{\sigma} \sim 0.1 \mathrm{~km} \mathrm{~s}^{-1}$, while the dispersion of the individual absorption components is $\Delta V_{ \pm} \sim 100 \mathrm{~km} \mathrm{~s}^{-1}$, at least $\sim 10^{6}$ components are required, a number far beyond that available in the present data sets. This argument, even though in a more complex formulation, should apply also in a multi-line approach. A smaller number of components may be required if the velocity pattern of the material changes smoothly with position. For illustration, we present here a reasoning based on the assumption that all blobs move in circular orbits, seen edge-on, in a potential consistent with the profile given in Eq. (5). In this case, at a given radius $R$, the orbital velocity and the gravitational redshift (expressed as a velocity) are given respectively by:

$$
\begin{aligned}
V_{\mathrm{orb}}^{2}= & 4 \pi G \rho_{\mathrm{NFW}} R_{\mathrm{NFW}}^{3} \\
& \left(\ln \left(1+R / R_{\mathrm{NFW}}\right) / R-1 /\left(R_{\mathrm{NFW}}+R\right)\right), \\
V_{\text {grav }}= & 4 \pi G \rho_{\mathrm{NFW}} R_{\mathrm{NFW}}^{3} \ln \left(1+R / R_{\mathrm{NFW}}\right) /(R c) .
\end{aligned}
$$

Let us also assume that, at $R=R_{\mathrm{NFW}}$, the ratio $V_{\text {grav }} / V_{\text {orb }}=$ $1.577 \sqrt{4 \pi G \rho_{\mathrm{NFW}}} R_{\mathrm{NFW}} / c$ is of the order of the observed ratio $\Delta V_{\sigma} / \Delta V_{ \pm} \sim 10^{-3}$.

We consider a path along a line of sight with impact parameter $d$ with respect to the centre of the system: $R=\sqrt{l^{2}+d^{2}}$, where $l$ is the coordinate along the line of sight. We then compute $V_{\text {grav }}$ and the radial component of $V_{\text {orb }}\left(V_{\text {orb,r }}\right)$. Results are displayed in Fig. 5 for different values of $d$. While by a pure dimensional argument one would always expect values of the 


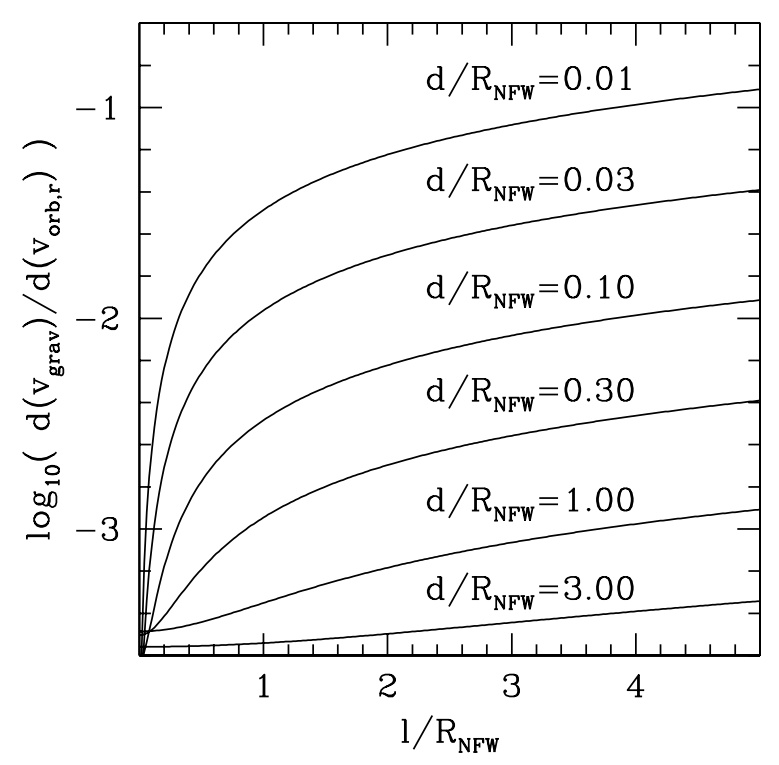

Fig. 5. Relative change of $V_{\text {grav }}$ and $V_{\text {orb,r }}$, along the line of sight, for different values of the impact parameter $d$.

order of $\Delta V_{\sigma} / \Delta V_{ \pm} \sim 10^{-3}$, Fig. 5 shows that there may be conditions in which this ratio can be one, or even two orders of magnitude higher. In addition, in the case of orbits tilted by an angle $\zeta$, a further $1 / \cos (\zeta)$ factor should be included. This shows that, under favorable conditions, a sample of $\sim 100$ components, spread over a set of absorption line systems, may be sufficient to get a statistically significant detection of GRS.

\subsection{Component misidentification: How GRS breaks the symmetry}

MFW aim at comparing components, in different transitions, which are attributed to the same cloud, i.e. to the same physical region within the absorption system. Of course, if this goal is reached, no GRS would be detected. Let us examine instead the case of "component misidentification", namely the case in which two components, identified in the line profile of two or more different transitions, are erroneously attributed to the same cloud. There may be different reasons why a given transition effectively samples inner region clouds compared to another transition. This may happen, for instance, if chemical or ionization radial gradients are present in the absorption system. There is some evidence of chemical and ionization inhomogeneities in high column density absorbers, for example, which are very numerous in our high- $z$ sample (Petitjean \& Srianand 1999; Petitjean et al. 2002). But even in the absence of such gradients, inner regions are usually denser than outer regions, and absorption lines of different relative optical depth saturate at different distances from the centre and lines. For this reason, lines with different $\tau$ might effectively sample different regions in the potential well, and therefore may be subject to different levels of gravitational redshift. From this scenario it naturally follows that one should find a correlation between $\Delta v / v$ and $\tau$ for different transitions, and therefore a correlation between $\Delta \alpha / \alpha$ and $\lambda$ (see Sect. 2). Therefore our working hypothesis of Sect. 2 (a primary correlation between $\Delta v / v$ and $\tau$ ) is derived naturally from the idea that GRS and component misidentification may play a role in some determination of $\Delta \alpha / \alpha$. In our framework, if transitions of different relative optical depths sample different regions of an absorption system, high-contrast absorption systems should in fact be the most appropriate ones to reveal effects of differential gravitational redshift. The correlations we discuss in Sect. 2.2 indicate indeed that strong systematic effects are present in this subsample.

In the presence of component misidentification, if clouds seen in absorption in two different transitions are typically located at different distances from the centre, their frequency ratio is affected by the different radial velocities as well as by the different halo gravitational potential felt by the two clouds. Symmetry arguments imply that spurious deviations caused by the different kinematic properties of the clouds average to zero in a suitably large sample. Therefore only GRS can reasonably account for any residual systematic shift in the average value of $\Delta \alpha / \alpha$.

\section{Correlations between line frequency shifts and gravitational potential}

In Sect. 2 we have shown that the concept of $\lambda$ is very effective in searching for hidden correlations. This does not necessarily imply that GRS plays a role in generating systematic effects, even though some working hypotheses suggest that it might. In this section we assume that, for a uniform sample in $\lambda$, the derived value of $\Delta \alpha / \alpha$ is roughly proportional to the level of GRS. Since GRS is a function of the galactic potential, we investigate whether $\Delta \alpha / \alpha$ correlates with indicators of the gravitational potential strength. The gravitational potential depends on the total mass associated with the absorption system and on the radial distance of the absorbing material from the centre of mass, but unfortunately none of these physical quantities is directly measurable from absorption data. A way out is to identify observable quantities, which correlate with total mass and radius.

A quantity that can correlate with the strength of the gravitational potential is the metal column density. In fact, for constant metal abundances, more massive absorption systems have higher gas column density (and therefore higher metal column densities). In addition, metals are expected to be overabundant towards the centre of the potential well or in more massive systems because of the longer star formation history.

In this section we examine two samples of objects, both with known Fe II column densities (hereafter $N(\mathrm{Fe}$ II )). The first one contains low- $z$ absorption systems (as listed in Table 3 of Murphy et al. 2003a) that have well documented N(Fe II ) along the line of sight. Most of these objects have been analyzed by Churchill (1997, C97), or by Churchill \& Vogt (2001, CV01). In order to obtain a uniform sample, we have limited our selection to these two references. The resulting sample contains 24 absorbing systems at redshifts $0.5<z_{\text {abs }}<1.8$, and their $\log N(\mathrm{Fe}$ II $)$ is listed in Table 3 . This sample is statistically consistent with the larger low- $z$ sample analyzed in the previous section. Our second sample contains high- $z$, damped Ly $\alpha$ absorption systems (with $N(\mathrm{HI}) \geq 10^{20} \mathrm{~cm}^{-2}$ and $z_{\mathrm{abs}}<z_{\mathrm{em}}$ ). In order to have a nearly uniform sample we select 22 of them 
Table 3. Fe II -selected absorption line systems.

\begin{tabular}{|c|c|c|c|c|}
\hline QSO & $z_{\mathrm{abs}}$ & $\Delta \alpha / \alpha\left(10^{-5}\right)$ & $\begin{array}{c}\log N(\mathrm{Fe} \text { II }) \\
\mathrm{cm}^{-2}\end{array}$ & Ref. \\
\hline $0002+05$ & 0.851 & $-0.346 \pm 1.279$ & 13.802 & (CV01) \\
\hline $0117+21$ & 0.729 & $0.084 \pm 1.297$ & 12.839 & (C97) \\
\hline $0117+21$ & 1.048 & $-0.223 \pm 2.200$ & 12.718 & (CV01) \\
\hline $0117+21$ & 1.343 & $-1.290 \pm 0.948$ & 12.484 & (C97) \\
\hline 0420-01 & 0.633 & $4.211 \pm 4.076$ & 12.879 & (CV01) \\
\hline 0450-13 & 1.175 & $-3.070 \pm 1.098$ & 14.985 & (C97) \\
\hline $0454+03$ & 0.860 & $0.405 \pm 1.325$ & 15.068 & (CV01) \\
\hline $0454+03$ & 1.153 & $-0.749 \pm 1.782$ & 12.826 & (CV01) \\
\hline $0823-22$ & 0.911 & $-0.394 \pm 0.609$ & 13.597 & (CV01) \\
\hline $1148+38$ & 0.553 & $-1.861 \pm 1.716$ & 13.186 & (CV01) \\
\hline $1206+45$ & 0.928 & $-0.218 \pm 1.389$ & 12.905 & (CV01) \\
\hline $1213-00$ & 1.320 & $-0.738 \pm 0.760$ & 14.529 & (C97) \\
\hline $1213-00$ & 1.554 & $-1.268 \pm 0.892$ & 14.404 & (C97) \\
\hline $1222+22$ & 0.668 & $0.067 \pm 1.474$ & 13.115 & (CV01) \\
\hline $1225+31$ & 1.795 & $-1.296 \pm 1.049$ & 13.881 & (C97) \\
\hline $1248+40$ & 0.773 & $2.165 \pm 1.191$ & 13.550 & (CV01) \\
\hline $1248+40$ & 0.855 & $-0.021 \pm 1.268$ & 12.533 & (C97) \\
\hline $1254+04$ & 0.519 & $-3.371 \pm 3.247$ & 13.957 & (CV01) \\
\hline $1254+04$ & 0.934 & $1.485 \pm 1.908$ & 12.699 & (CV01) \\
\hline $1317+27$ & 0.660 & $0.590 \pm 1.515$ & 13.100 & (CV01) \\
\hline $1421+33$ & 0.843 & $0.099 \pm 0.847$ & 13.143 & (C97) \\
\hline $1421+33$ & 0.903 & $-0.998 \pm 1.783$ & 13.706 & (CV01) \\
\hline $1421+33$ & 1.173 & $-2.844 \pm 1.448$ & 13.049 & (CV01) \\
\hline $1634+70$ & 0.990 & $1.094 \pm 2.459$ & 13.059 & (CV01) \\
\hline $0000-26$ & 3.390 & $-7.666 \pm$ & 1 & (PW99) \\
\hline 0019-15 & 3.439 & $0.925 \pm 3.958$ & 14.770 & (PW99) \\
\hline $0100+13$ & 2.310 & $-3.941 \pm 1.368$ & 15.096 & (PW99) \\
\hline $0149+33$ & 2.140 & $-5.112 \pm 2.118$ & 14.202 & (PW99) \\
\hline $0201+37$ & 2.462 & $0.572 \pm 1.719$ & 15.060 & (PW96) \\
\hline $0216+08$ & 1.768 & $0.044 \pm 1.235$ & 14.530 & (L96) \\
\hline 0347-38 & 3.025 & $-2.795 \pm 3.429$ & 14.503 & (P01) \\
\hline $0528-25$ & 2.141 & $-0.853 \pm 0.880$ & 14.940 & (L96) \\
\hline $0741+47$ & 3.017 & $0.794 \pm 1.796$ & 14.041 & (P01) \\
\hline $0841+12$ & 2.476 & $-4.304 \pm 1.944$ & 14.434 & (PW99) \\
\hline $1215+33$ & 1.999 & $5.648 \pm 3.131$ & 14.648 & (PW99) \\
\hline $1425+60$ & 2.827 & $0.433 \pm 0.827$ & 14.480 & (L96) \\
\hline $1759+75$ & 2.625 & $-0.750 \pm 1.387$ & 15.091 & (P01) \\
\hline $1946+76$ & 2.843 & $-4.743 \pm 1.289$ & 13.380 & (L96) \\
\hline $2206-20$ & 1.920 & $1.878 \pm 0.702$ & 15.458 & (PW97) \\
\hline $2206-20$ & 2.076 & $1.429 \pm 3.022$ & 13.320 & (PW97) \\
\hline $2230+02$ & 1.864 & $-0.998 \pm 0.492$ & 15.188 & (PW99) \\
\hline $2231-00$ & 2.066 & $-2.604 \pm 1.015$ & 14.750 & (PW99) \\
\hline $2348-14$ & 2.279 & $1.346 \pm 4.180$ & 13.792 & (PW99) \\
\hline $2359-02$ & 2.095 & $-0.068 \pm 0.722$ & 14.507 & (PW99) \\
\hline 2359-02 & 2.154 & $4.346 \pm 3.338$ & 13.895 & (PW99) \\
\hline
\end{tabular}

(C97) Churchill (1997); (CV01) Churchill \& Vogt (2001); (L96) Lu et al. (1996); (PW96) Prochaska \& Wolfe (1996); (PW97) Prochaska \& Wolfe (1997); (PW99) Prochaska \& Wolfe (1999); (P01) Prochaska et al. (2001).

with $1.8 \leq z_{\mathrm{abs}}<3.5$, and published values of $\log N(\mathrm{Fe}$ II $)$ in at least one of the following references: (L96) Lu et al. (1996), (PW96) Prochaska \& Wolfe (1996), (PW97) Prochaska \& Wolfe (1997), (PW99) Prochaska \& Wolfe (1999), (P01)
Prochaska et al. (2001). The sample is listed in the last 21 entries of Table 3 .

In order to estimate the dependence of the measured values of $\Delta \alpha / \alpha$ on $N(\mathrm{Fe}$ II $)$, we will use a linear regression, adopting the functional dependence $\Delta \alpha / \alpha=m(\log N(\mathrm{Fe}$ II $)-13.5)+q$. In this way, for the whole low- $z$ sample of Table 3 , we obtain $m=(-0.45 \pm 0.33) \times 10^{-5}$ and $q=(-0.50 \pm 0.25) \times 10^{-5}$, with a reduced $\chi^{2}$ of 0.90 : the value obtained for the slope $m$ is not statistically significant $(1.4 \sigma)$. For the high- $z$ sample of Table 3, instead, we calculate $m=(1.19 \pm 0.51) \times 10^{-5}$ and $q=(-2.21 \pm 0.72) \times 10^{-5}$, with a reduced $\chi^{2}$ of 2.65 : a reasonable $(2.3 \sigma)$ significance level is obtained here, but the reduced $\chi^{2}$ is anomalously high. We attribute this result to the fact that some of the quoted uncertainties on $\Delta \alpha / \alpha$, which are relative to high-contrast damped systems, are underestimated (see Fig. $3 \mathrm{a}$, and the discussion in the previous section). In fact, if we compute a linear regression on the high- $z$ sample, assigning the same uncertainty to all measurements of $\Delta \alpha / \alpha$, we obtain $m=(-0.38 \pm 1.20) \times 10^{-5}$ and $q=(-0.27 \pm 1.45) \times 10^{-5}$ : therefore, also in this case the slope $m$ is not statistically significant. We attribute the low significance of these correlations to the fact that none of the two samples is uniform in $\lambda$.

Luckily, 18 out of the 24 objects of the low- $z$ sample lie in a narrow range of $\lambda$, namely $(-0.92,-0.79)$. The correlation becomes more significant if we select only absorbers with high $\log N(\mathrm{Fe}$ II $)$ values in this $\lambda$ range. Taking $\log N(\mathrm{Fe}$ II $)>13.0$ (12 objects) we obtain $m=(-1.23 \pm 0.56) \times 10^{-5}(2.2 \sigma)$ and $q=(-0.35 \pm 0.34) \times 10^{-5}$, with a reduced $\chi^{2}$ of 1.14 . For $\log N($ Fe II $)>13.5(6$ objects $) m=(-2.16 \pm 0.78) \times 10^{-5}$ $(2.8 \sigma)$ and $q=(0.34 \pm 0.53) \times 10^{-5}$, with a reduced $\chi^{2}$ of 0.92 . This result is consistent with a $\Delta \alpha / \alpha$ that deviates significantly from zero only for $\log N(\mathrm{Fe}$ II $)$ larger than about 13.5. The $\Delta \alpha / \alpha$ deviation is towards more negative values as $\log N(\mathrm{Fe}$ II $)$ increases. Although the statistical significance is not high (only $2.8 \sigma$ in the best case), one should notice that negative deviations of $\Delta \alpha / \alpha$ for negative $\lambda$ are consistent with the increase of $\Delta \alpha / \alpha$ with increasing $\lambda$, obtained in the previous section for the low- $z$ sample. This can be explained in terms of an effective line segregation, with weaker lines sampling effectively regions closer to the centre of the potential well. GRS is a reasonable candidate to explain the decrease in $\Delta \alpha / \alpha$ with increasing $\log N(\mathrm{Fe}$ II $)$, because systems with higher metal column density are either closer to the centre of the potential well, or located in more massive systems or both.

There have been other proposals to explain the VFSC result. Of particular interest is the consistency of the VFSC values with a non-solar isotopic ratio of $\left({ }^{25} \mathrm{Mg}+{ }^{26} \mathrm{Mg}\right) / 24 \mathrm{Mg}$ occurring at large redshifts. If this isotopic ratio increases at large redshifts, small apparent shifts would be introduced in the absorption lines (Ashenfelter et al. 2004). These shifts mimic a VFSC if instead a solar isotopic ratio of magnesium is used, as in the MWF analysis. The large uncertainty about this proposal lies in the magnesium isotopic ratio variations with metallicity. Ashenfelter et al. (2004) show that an Initial Mass Function (IMF) particularly rich in intermediate mass stars is needed for an increase of the magnesium isotopic ratio with decreasing metallicity (in the range $[\mathrm{Fe} / \mathrm{H}]=0,-1.5$ ). Analyses of low metallicity star data (Gay \& Lambert 2000) show instead an 
opposite trend of the magnesium isotopic ratio variations with metallicity. If the data by Gay \& Lambert (2000) reflect the situation in QSO absorbers, which have lower metallicity than solar, then magnesium isotopic ratio variations cannot explain the negative $\Delta \alpha / \alpha$ reported by MWF. In this case metallicities lower than solar would correspond to lower magnesium isotopic ratios and to positive value of $\Delta \alpha / \alpha$. If one assumes instead that the results of Ashenfelter et al. (2004) apply to gas in QSO absorbers, then magnesium isotopic ratio variations can explain the MWF results. The correlation between $\Delta \alpha / \alpha$ and metallicity should in this case be positive for a certain range of $[\mathrm{Fe} / \mathrm{H}]$ (which depends on IMF and yields). For the GRS hypothesis instead large variations of $\Delta \alpha / \alpha$ should be associated with large potential wells. Since more massive galaxies have had a longer star formation history, a negative correlation between metallicity and $\Delta \alpha / \alpha$ should appear. The results of this Section seem to favour the GRS hypothesis, but accurate metal abundances, higher significance in the outlined correlations and more information on earlier IMF are needed to draw any conclusions on the magnesium isotopic ratio variation hypothesis.

\section{Conclusions}

Even though we cannot disprove completely the VFSC hypothesis of Murphy et al. (2003a), in this paper we have discovered systematic effects which can mimic a non-zero $\Delta \alpha / \alpha$. Often $\Delta \alpha / \alpha$ depends on $\lambda$, the correlation coefficient between two atomic quantities: the relative optical depth and the relativistic correction coefficient $Q$. For the atomic transitions used in the MWF analysis for each absorber, we evaluate $\lambda$ and notice that the $\lambda$ distribution is not symmetric around zero: this justifies the net displacement of the average $\Delta \alpha / \alpha$ from zero. In particular, the correlation between spectral shifts and the relative optical depth of the various transitions seems to be the origin of correlations between spectral shifts and $Q$, used by MWF as evidence for a VFSC. Non-zero values of $\Delta \alpha / \alpha$ may then be the result of GRS if lines of different relative optical depth effectively sample regions at different distances from the centre of the potential well.

We have examined the low $-z$ and the high- $z$ samples of Murphy et al. (2003a) separately, since they have been analyzed in different sets of transitions and furthermore are associated with different types of cosmic structures (see Sect. 2 for more details). In the low- $z$ sample the correlation between $\Delta \alpha / \alpha$ and $\lambda$ can be interpreted in terms of a primary positive correlation between $\Delta v / v$ and the relative optical depth: that means that relatively weaker lines are more redshifted than others (i.e. $\Delta v$ more negative). This is in agreement with what one would expect from GRS, under the hypothesis that intrinsically weaker lines are visible only through dense regions, located closer to the centre of the absorption system. Since for this sample most of the systems lie in a restricted $\lambda$ range, and we know the metal column densities, we compare $N(\mathrm{Fe}$ II $)$ with the derived $\Delta \alpha / \alpha$ values. Systems with higher $N(\mathrm{Fe}$ II $)$ values have more negative $\Delta \alpha / \alpha$ values. This again confirms the GRS hypothesis since more negative $\Delta \alpha / \alpha$ can be interpreted as more heavily gravitationally redshifted lines. High $N(\mathrm{Fe}$ II ) values are in fact expected when the gas lies in regions of strong gravitational potential. If future data prove a positive correlation between $\Delta \alpha / \alpha$ and metal abundances, this will support GRSrelated studies and weaken other proposals, such as the magnesium isotopic ratio variations, for explaining the non-zero value of $\Delta \alpha / \alpha$ derived in the MWF analysis.

For the "high-contrast" subsample of the high- $z$ systems, for which Murphy et al. (2003a) have reported an anomalous statistical dispersion in $\Delta \alpha / \alpha$, we find instead a systematic effect that can be interpreted in terms of a primary inverse correlation between $\Delta v / v$ and the relative optical depth. Further dividing this subsample into heavily damped systems, with $N(\mathrm{HI})>N_{\mathrm{c}}$, and systems with $N(\mathrm{HI}) \leq N_{\mathrm{c}}$, we have discovered that the latter subsample shows a striking inverse correlation, at a level higher than $4.5 \sigma$ for $N_{\mathrm{c}} \leq 7 \times 10^{20} \mathrm{~cm}^{-2}$. No correlation is present for $N(\mathrm{HI})>N_{\mathrm{c}}$. So, while for the heavily damped systems the MWF interpretation of the anomalous statistical dispersion might be correct, for lower column density systems we believe that the anomalous statistical dispersion is indeed the effect of an underlying correlation.

Note that the sign of the correlation between $\Delta \alpha / \alpha$ and $\lambda$ for the high-contrast (high-z) sample is opposite to that obtained for the low- $z$ one. If this correlation is interpreted in terms of GRS, stronger transitions should be associated with innermost regions of the absorption system. This is opposite to what is inferred for the low- $z$ sample, and requires the presence of physical inhomogeneities within the system (as actually found in some cases. e.g. Petitjean \& Srianand 1999; Petitjean et al. 2002). However, one cannot exclude that the opposite trends simply arise because the bias comes out differently in the MWF procedure used to estimate $\Delta \alpha / \alpha$ : this is because the data and the characteristics of the absorption systems in the two samples are very different.

As already pointed out, a more detailed analysis can be carried out having access to the original data. For instance, it would be useful to obtain subsamples analyzed uniformly, using the same set of transitions. Conversely, one could also investigate how $\Delta \alpha / \alpha$ changes with different choices of the fitted transitions, in the same absorption system. More generally, instead of determining how $\Delta v / v$ correlates with $Q$ (from which $\Delta \alpha / \alpha$ is determined), one should search for correlations of $\Delta v / v$ with other quantities, such as the relative optical depth. Notice that any quoted variation of $\Delta \alpha / \alpha$ with cosmological time should be taken very cautiously, because different sets of transitions are used for different redshifts.

In a recent work, Chand et al. (2004) found no variation in the fine structure constant for a sample of low- $z$ absorption systems. They obtained this result avoiding both weak and saturated lines, as well as heavily blended spectral regions. In this way they restricted their analysis mostly to intermediate "satellite components", avoiding the ones relative to central or outermost regions. Therefore they reached a better accuracy in positioning the subcomponents. A contamination of GRS on $\Delta \alpha / \alpha$ is possible only in the presence of some bias in the spectral analysis. We do not find any significant correlation between $\Delta \alpha / \alpha$ and $\lambda$ in the Chand et al. (2004) results. Their work further supports the idea that biases may originate from the fit of weak spectral components or of the saturated and complex 
parts of a line structure. Moreover, GRS effects are harder to detect in a sample which avoids the centremost and outermost regions since this choice effectively restricts the range of the potential well tested.

We would like to encourage any future GRS experiment over cosmological distances since the results can be unique tools to test structure formation scenarios in the context of several cosmological models. For example if at redshifts as high as 4-5 the Universe is still dominated by small mass objects (Cold Dark Matter scenario), GRS-induced line frequency shifts in absorption systems at such redshifts should be smaller than the ones detectable at more recent times. Also theoretical models of possible variations of the fine structure constant, which take into account the observational state of the art are needed (see e.g. Steinhardt 2003), in order to check the consistency of different results.

Acknowledgements. We are grateful to the referee and to Charles Steinhardt for many valuable comments to the original version of this manuscript.

\section{References}

Ashenfelter, T., Mathews, G. J., \& Olive, K. A. 2004, ApJ, 615, 82 Avila-Reese, V., Colin, P., Valenzuela, O., D’Onghia, E., \& Firmani, C. 2001, ApJ, 559, 516

Bahcall, J. N., Steinhardt, C. L., \& Schlegel, D. 2004, ApJ, 600, 520

Broadhurst, T., \& Scannapieco, E. 2000, ApJ, 533, L93

Burkert, A. 1995, ApJ, 447, L25

Chand, H., Srianand, R., Petitjean, P., \& Aracil, B. 2004, A\&A, 417, 853

Churchill, C. W. 1997, Ph.D. Thesis, Univ. of California, Santa Cruz Churchill, C. W., \& Vogt, S. S. 2001, AJ, 122, 679

Curran, S. J., Webb, J. K., \& Murphy, M. T., et al. 2002, PASA, 19, 455

Dzuba, V. A., Flambaum, V. V., Kozlov, M. G., \& Marchenko, M. 2002, PhRvA, 66, 022501

Eke, V. R., Navarro, J. F., \& Steinmetz, M. 2001, ApJ, 554, 114

Gay, P. L., \& Lambert D. L. 2000, ApJ, 533, 260
Lanzetta, K. M., Wolfe, A. M., Turnshek, D. A., et al. 1991, ApJS, 77, 1

Lu, L., Sargent, W. L. W., Barlow, T. A., Churchill, C. W., Vogt, S. S. 1996, ApJS, 107, 475

Lu, L., Sargent, W. L. W., \& Barlow, T. A., 1998, AJ, 115, 55

Martins, C. J. A. P. 2003, Ap\&SS, Proc. of the JENAM 2002 Workshop The Cosmology of Extra Dimensions and Varying Fundamental Constants, 283

Murphy, M. T., Webb, J. K., Flambaum, V. V., et al. 2001a, MNRAS, 327, 1208

Murphy, M. T., Webb, J. K., Flambaum, V. V., Churchill, C. W., \& Prochaska, J. X. 2001b, MNRAS, 327, 1223

Murphy, M. T., Webb, J. K., Flambaum, V. V., Prochaska, J. X., \& Wolfe, A. M. 2001c, MNRAS, 327, 1237

Murphy, M. T., Webb, J. K., \& Flambaum, V. V. 2003a, MNRAS, 345, 609

Murphy, M. T., Webb, J. K., Flambaum, V. V., \& Curran, S. J. 2003b, Ap\&SS, 283, 577

Navarro, J. F., Frenk, C. S., \& White, S. D. M. 1996, ApJ, 462, 563

Navarro, J. F., Frenk, C. S., \& White, S. D. M. 1997, ApJ, 490, 493

Outram, P. J., Chaffee, F. H., \& Carswell, R. F. 1999, MNRAS, 310, 289

Petitjean, P., \& Srianand, R. 1999, A\&A, 345, 73

Petitjean, P., Srianand, R., \& Ledoux C. 2002, MNRAS, 332, 383

Prochaska, J. X., \& Wolfe, A. M. 1996, ApJ, 470, 403

Prochaska, J. X., \& Wolfe, A. M. 1997, ApJ, 474, 140

Prochaska, J. X., Wolfe, A. M. 1999, ApJS, 121, 369

Prochaska, J. X., Wolfe, A. M., Tytler, D., et al. 2001, ApJS, 137, 21

Steinhardt, C. L. 2003, PhRvD, submitted, [arXiv:hep-ph/0308253]

Stiavelli, M., \& Setti, G. 1993, MNRAS, 262, L51

Uzan, J.-P. 2003, RvMP, 75, 403

Uzan, J.-P. 2004, in The Quest for Cosmological Scalar Fields, in press [arXiv: astro-ph/0409424]

Webb, J. K., Flambaum, V. V., Churchill, C. W., Drinkwater, M. J., \& Barrow, J. D. 1999, PhRvL, 82, 884

Webb, J. K., Murphy, M. T., Flambaum, V. V., et al. 2001, PhRvL, 87, 091301

Webb, J. K., Murphy, M. T., Flambaum, V. V., \& Curran, S. J. 2003, Ap\&SS, 283, 565 